\title{
PREVALENCE OF SARCOPENIA IN ELDERLY WITH DEMENTIA INSTITUTIONALIZED: A MULTICENTER STUDY
}

\author{
C.M. Sarabia Cobo' , V. Pérez², C. Hermosilla², M.J. Nuñez'2, P. de Lorena²
}

\begin{abstract}
Objective: Determine the prevalence of sarcopenia, applying the new diagnostic criteria in a group of institutionalized elderly with dementia, analyzing the possible association between sarcopenia, degree of dementia, and nutritional status. Method: Transversal, descriptive, multicenter study with 189 subjects diagnosed with dementia in middle phase. The current criteria for sarcopenia were assessed through several tests (bioimpedance, dynamometry, and physical performance tests). Nutritional status through the Mini Nutritional Assessment (MNA) was also assessed. Parametric tests (Student $t$ test and ANOVA) were performed to compare the significant differences between the two stages of dementia according to gender, age, presence of sarcopenia, and MNA, among other tests. Results: $74 \%$ were female and the mean age was 82.3 years. Of the participants, $57.1 \%$ were in stage 5 and $42.9 \%$ in stage 6 in GDS. The MNA showed that $54.6 \%$ had risk of malnutrition, $36.2 \%$ were normal, and $9.2 \%$ had malnutrition. Of the subjects, $68.78 \%$ have shown signs of sarcopenia. There were significant differences for sarcopenia according to the phase of dementia and between sarcopenia and malnutrition status. Conclusions: To our knowledge it is the first study of its kind in Spain under the new criteria for diagnosis of sarcopenia. There seems to be a high percentage of sarcopenia among institutionalized elderly with dementia and this in turn appears to be related to dementia status and states of malnutrition. This syndrome has a significant impact on the population, so early detection is crucial; more studies of this type are required.
\end{abstract}

Key words: Sarcopenia, malnutrition, dementia, nutritional assessment, older.

\section{Introduction}

The World Health Organization (WHO), in its latest study on nutrition in aging, identified the elderly population as a nutritionally vulnerable group, due to their own anatomical and physiological aging changes process (1).

Among changes brought about by the aging process is the loss of muscle mass and strength and decreased physical function. The loss of muscle mass associated with aging is called primary sarcopenia (2). The term sarcopenia has undergone several definitions (3) with no clear consensus on this issue; as a result the European Working Group on Sarcopenia in Older People (EWGSOP) developed consensus diagnostic criteria of age-related sarcopenia in 2010 (4). Sarcopenia is defined as "a syndrome characterized by a progressive and generalized loss of skeletal muscle mass and strength with risk for adverse outcomes such as physical disability, poor quality of life and mortality." The

1. Cantabria University, Spain; 2. CR Santa Lucía, Spain

Corresponding Author: Sarabia-Cobo Carmen María, Ph.D, RN, MSN, Professor of Gerontological Nursing, Nursing School. Cantabria University, Spain, Avda. Valdecilla s/n 39011, Santander, Cantabria, Spain, +34 9422022 39, carmen.sarabia@unican.es confirmation EWGSOP establishes several criteria for diagnosing sarcopenia: low muscle mass, loss of muscle strength, and reduced physical performance. Sarcopenia should be associated with at least the first one and one of the other two.

Regarding the prevalence, data are not conclusive because of the lack of consensus criteria listed above (5, 6). Studies estimate that this varies from 3 to $30 \%$ depending on the variables used to establish the diagnosis (6-9). Estimates of the importance of their presence lie in the negative effect of sarcopenia on the autonomy of the elderly, significantly increasing the risk of falls and other complications (10). This causes an increase in morbidity and vulnerability of this group.

Given that the studies are not conclusive as to the etiology or the diagnosis criteria, (11) need further research in this regard especially as this is a very prevalent disease for the elderly population and leads to increased vulnerability and fragility, such as dementia.

The overall prevalence of dementia is currently estimated at 24 million; studies show that the figure will double by 2040 (12). Alzheimer's disease (AD) is the most common primary dementia. It's characterized by progressive cognitive decline, is irreversible, and no treatment currently exists. The AD has several phases in 
which patients increase their dependence and need for care by others. The terminal phase of the disease results in prostration, with patients becoming bedridden and totally dependent. Studies show that the nutritional status of institutionalized patients with $\mathrm{AD}$ is worse than those who are also institutionalized at the same age but have no dementia $(13,14)$.

So far there are few prevalence studies applying the new diagnostic criteria for sarcopenia and even fewer among the institutionalized elderly with dementia. The main objective of this study was to determine the prevalence of sarcopenia, applying the criteria and the diagnostic algorithm proposed by EWGSOP, in a group of institutionalized elderly with $\mathrm{AD}$ in seven senior centers in different parts of Spain, analyzing the possible association between sarcopenia, degree of dementia, and nutritional status.

\section{Material and methods}

Transversal, descriptive and multicenter study. The sample consisted of 189 subjects diagnosed with probable $\mathrm{AD}$ (by the relevant departments of neurology) at seven residential centers located in seven cities in Spain (belonging to the same foundation), seniors 65 years or older, of both sexes. Those subjects were selected in evolutionary stages means (levels 5 and 6 of the Global Deterioration Scale scales) and Functional Assessment Staging (GDS and FAST, respectively) $(15,16)$.

For the diagnosis of sarcopenia, following the criteria of EWGSOP, mass and muscle strength and physical performance should be evaluated. According to the same EWGSOP, all parameters to measure the three necessary criteria were selected for this study: (a) analysis of bioimpedance to mediate muscle mass (discarding anthropometric measurements because the report "Anthropometric measurements are vulnerable to error and not recommended for routine use in the diagnosis of sarcopenia" ) being used appliance model Tanita BC- 418 (B; (b) force manual to measure pressure by manual muscle strength dynamometer (Takei TKK digital dynamometer model $5401{ }^{\circledR}$ - range 5-100 kg ), carrying two alternative attempts with each hand in a standardized position, standing if possible, with arms parallel to the body without contact; (c) series of short physical performances (SPPB) to measure physical performance.

The nutritional status of subjects were also assessed through the scale Mini Nutritional Assessment (MNA), with three possible diagnoses: Normality, Risk of malnutrition and Malnutrition. This test is usually harvested in the clinical history of the residents within the Comprehensive Geriatric Assessment (GA) and is reassessed every three months in patients with dementia. There is a specific protocol for the preparation of the Integral Geriatric Value and MNA which is followed by all study centers.

Data collection was performed by the nursing staff of the centers during September and October 2013.

Consent was obtained to conduct the study: the general management of the foundation, residential centers, the ethics committee attached to the main residential center (in Madrid), and all participants and/or guardians were properly informed.

The statistical treatment of the data was performed with SPSS 19.0 software, conducted a descriptive analysis and a frequency depending on each variable. Although you can assume normal distribution for the number of subjects $(\mathrm{N}>30)$ Kolmogorov- Smirnov (KS) was performed to examine the normality and the Levene test to assess the homogeneity of variance. Significance tests were performed using Chi square test $\left(\mathrm{X}^{2}\right)$ to evaluate possible differences between nominal variables (the evolutionary stage of dementia by sex, diagnosis of sarcopenia by sex, dementia status, and the presence of sarcopenia) and Student t (when parametric criteria were met) to assess the stage of dementia according to age, diagnosis of sarcopenia and age, and ANOVA to assess association between stage of dementia and MNA, and between the presence of sarcopenia and stage MNA $(\alpha=$ 0.05 in all cases).

\section{Results}

Of the 189 cases, $74 \%$ were women, the mean age was 82.3 years (95\% CI: 77.1 to 83.60 , range: $69-101$ ). $57.1 \%$ were in stage 5 and $42.9 \%$ in stage 6 GDS.

The KS test indicated normality $(\mathrm{KS}=0.083, \mathrm{p}=0.200)$ and Levene indicated homoscedasticity (17.27, $\mathrm{p}=0.09)$.

No significant differences between the evolutionary stage of dementia was found by $\operatorname{sex}\left(X^{2}=0.687, p=0.123\right)$ or age $(\mathrm{t}=0.541, \mathrm{p}=0,214)$. MNA results indicated that $54.6 \%$ of the sample had risk of malnutrition, $36.2 \%$ were normal, and $9.2 \%$ had malnutrition. There were no significant differences in response to the phase of dementia.

Sarcopenia was diagnosed in $68.78 \%$ of patients, of whom $47.8 \%$ met all three criteria and $31.2 \%$ met two criteria (would be considered mild sarcopenia). There was no significant differences in the diagnosis of sarcopenia by sex, but there was by age $\left(x^{2}=0.325, p=\right.$ $0.00)$.

Analyses indicated that depending on the stage of dementia weren't found significant differences for the MNA $(\mathrm{F}=123.2, \mathrm{p}>0.05)$ but were found for the presence of sarcopenia $(\mathrm{X} 2=0.852, \mathrm{p}=0.00)$. Significant differences between sarcopenia and malnutrition status were also found $(F=0.556, p=0.01)$, but not for the Risk of Malnutrition status and Normality status.

Table 1 shows the most relevant results of bioimpedance (Fat Mass -FM- and Fat Free Mass -FFM-) is based on the diagnosis of sarcopenia, appreciating that 
there are no significant differences. Also no significant differences between the percentages of FM and FFM depending on the stage of EA were found.

Table 1

Percentage of FM and FFM in relation with sarcopenia diagnosis

\begin{tabular}{lccc}
\hline & No Sarcopenia & Sarcopenia & $\mathbf{P}^{*}$ \\
\hline FM $(\%)$ & 34,8 & 31,8 & 0,127 \\
FFM $(\%)$ & 65,2 & 68,2 & 0,109 \\
\hline
\end{tabular}

FM Fat Mass; FFM Fat Free Mass; * $\mathrm{X}^{2}$

\section{Discussion}

The average profile of the sample was that of a woman of 82 years and stage 5 GDS/FAST AD. No significant differences by age or sex, depending on the type of stage which coincides with the literature when listing these phases, were found to dementia (12). Regarding nutritional status, we know that MNA is a validated and widely used instrument in the geriatric (17) population and older with dementia as indicated by a study of Walk et al., in which the authors indicated a good correlation between MNA and GDS stage/FAST dementia (18). However it should be noted that people with cognitive impairment often respond to caregivers, but this instrument is incomplete at least partially biased to pick a section of subjective perception in patients with dementia. Yet the results found in this study are similar to others conducted in institutionalized persons with dementia $(19,20)$.

The high percentage of sarcopenia found $(68.78 \%)$ is very striking compared with other recent research on the population (healthy most of the time without disease or cognitive impairment) (5-9). There is a study in Spain in the hospitalized older population $(n=205)$ having an even slightly higher $(76.4 \%)$ (21) similar percentage. To our knowledge there are no studies that have examined the prevalence of sarcopenia in institutionalized elders with dementia following the latest guidelines of the EWGSOP, so there is no data with which to compare these findings. However, it is feasible that the high prevalence relates to two factors that may be associated with sarcopenia, such as weight loss and widely studied malnutrition associated with dementia, although the ethiopatogeny is not clearly understood (14, 20-22). If you add to this that the results show a statistically significant association between malnutrition and Sarcopenia and between sarcopenia and dementia we could speculate that dementia can be considered in itself a major risk factor for developing sarcopenia. However, the data should be taken with caution as they also found a significant association between sarcopenia and age that is consistent with the literature and with the definition of sarcopenia $(4,11)$, although, as already mentioned, the prevalence in the population is much smaller than in our study $(5,9)$ in our study. We could also speculate that the MNA, though incomplete to measure the nutritional status of these patients, could be considered a predictor of sarcopenia, given the association found in our study.

It is also noteworthy that the authors decided to not include anthropometric data such as BMI or skinfolds in the studio, something quite unusual in comparison with such investigations (14, 20-22). This decision was made following the recommendations of EWGSOP indicating that these measures are not the most reliable in the diagnosis of sarcopenia (4). Therefore it is considered that analysis of this data was not the subject of this study (although they are calculated in the MNA and are required for bioimpedance), the most relevant being the MNA and bioimpedance measures as recommended to be more exact (23).

In this line, we found that measures of FM and FFM were constant regardless of the stage of dementia. This may be consistent with studies that advocate greater involvement of the FFM suggesting possible weight loss of these patients (which has not been evaluated as such in our study) is not solely due to deficits in intake, but would include metabolic and physical changes that occur in old age and seem to intensify in states of dementia (19, 24).

As for future research, the limitations of this study highlight that it would be desirable to increase the sample of patients with dementia to establish more specific diagnostic criteria for sarcopenia in this population. It is also necessary to perform studies similar to this, which we know is the first to determine the prevalence of sarcopenia in institutionalized AD patients. It would be advisable to carry out comparative studies with other populations with cognitive impairment (dementia in the community, Parkinson's disease, mild cognitive impairment, etc.).

It would also be appropriate in a future continuation of this study to include measures of BMI and plicometry to establish the prevalence of a diagnosis of malnutrition called sarcopenic obesity, a diagnosis that goes unnoticed, to determine the nutritional status of these patients (19). A 2012 study indicates a prevalence of 15\% in Spanish populations living in the community (25). In light of our data, presumably in the institutionalized elderly population with dementia, the prevalence is higher. Future research should also include the study of anthropometric measures to relate to sarcopenia, as those measures are easy to apply (the cheapest one is bioimpedance, for example) and several studies argue that they are suitable at least for screening for malnutrition $(26,27)$. Moreover also in the minds of the authors a future research should investigate the possibility of the significant association of two diagnostic 
criteria for sarcopenia (in our study were muscle strength assessed by dynamometry and physical activity through SPPB) as predictors of sarcopenia.

We can also indicate a limitation to address in future research. Having focused the study on the intermediate stages of dementia, it would be interesting to extend the sample upstream and downstream of the disease to compare the prevalence of sarcopenia in terms of phase dementia and as other studies suggest (19). This would add another research proposal to be a longitudinal study of a cohort of subjects to determine the evolution of sarcopenia and nutritional status of the individual along with dementia.

From all this we can conclude that studies should be maintained along this line, based on the agreed EWGSOP criteria, because sarcopenia has important implications for the health of the individual without even knowing pathogenesis. Because of the relevance and concern about the issue, the Spanish Society of Geriatrics and Gerontology has recently created an Observatory Sarcopenia (28). It is therefore crucial to continue delimiting the diagnostic criteria and include for this: evidence reliable, easy to apply in daily practice and that are appropriate for these patients according to their overall dependency and progressive deterioration.

Declaration of Conflicting Interests: The authors declared no potential conflicts of interest with respect to the research, authorship, and/or publication of this article.

Funding: The authors received no financial support for the research, authorship, and/or publication of this article.

\section{References}

1. OMS. Keep fit for life. Meeting the nutricional needs of older persons. Geneva, 2002.

2. Doherty TJ. Invited review: Aging and sarcopenia. J Appl Physiol 2003;95:1717-27

3. Rosenberg IH. Sarcopenia: origins and clinical relevance. J Nutr 1997;127: 990S-91S

4. Cruz-Jentoft AJ, Baeyens JP, Bauer JM, Boirie Y, Cederholm T, Landi F Zamboni $\mathrm{M}$ et al. Sarcopenia: European consensus on definition and diagnosis Report of the European Working Group on Sarcopenia in Older People. Age and ageing 2010;39(4): 412-23

5. Masanes F, Culla A, Navarro-Gonzalez M et al. Prevalence of sarcopenia in healthy community-dwelling elderly in an urban area of Barcelona (Spain). J Nutr Health Aging 2012;16:184-7

6. Baumgartner RN, Koehler KM, Gallagher D et al. Epidemiology of sarcopenia among the elderly in New Mexico. Am J Epidemiol 1998;147: 75563
7. Castillo EM, Goodman-Gruen D, Kritz-Silverstein D et al. Sarcopenia in elderly men and women: the Rancho Bernardo study. Am J Prev Med 2003;25: $226-31$

8. Chien MY, Huang TY, Wu YT. Prevalence of sarcopenia estimated using a bioelectrical impedance analysis prediction equation in community-dwelling elderly people in Taiwan. J Am Geriatr Soc 2008;56: 1710-5

9. Melton LJ III, Khosla S, Crowson CS et al. Epidemiology of sarcopenia. J Am Geriatr Soc 2000;48: 625-30

10. Inouye SK, Studenski S, Tinetti ME et al. Geriatric syndromes:clinical, research, and policy implications of a core geriatric concept. J Am Geriatr Soc 2007:55: 780-91

11. Cruz-Jentoft A, Landi F, Topinková E et al. Understanding sarcopenia as a geriatric syndrome. Curr Opin Clin Nutr Metab Care 2010;13: 1-7

12. Reitz C, Brayne C, Mayeux R. Epidemiology of Alzheimer disease. Natu Rev Neur 2011;7: 137-52

13. Shah R. The Role of Nutrition and Diet in Alzheimer Disease: A Systematic Review. J Amer Medi Dire Ass 2013;14(6): 398-402

14. Shatenstein B, Ferland G, Belleville S, Gray Donald K, Kergoat MJ, Morais J, et al. Diet quality and cognition among older adults from the NuAge study. Exp Gerontol 2012;47(5): 353-360

15. Reisberg B, Ferris SH, De León MJ, Crook T. The Global Deterioration Scale for assessment of primary degenerative dementia. Am J Psychiatry 1982;139 (9): 1136-9

16. Reisberg B. Functional assessment stating (FAST). Psychopharmacol Bull 1988;24(4): 653-9

17. Bauer JM, Volkert D, Wirth EM, et al. Diagnosing malnutrition in the elderly. Dtsch Med Wochenschr 2006;131(5): 223-7

18. Tarazona FJ, Belenguer A, Doménech JR, et al. Validez de la escala MNA como factor de riesgo nutricional en pacientes geriátricos institucionalizados con deterioro cognitivo moderado y severo. Nutr Hosp 24 (2009;6): 724-31

19. Camina M, de Mateo B, Carreño L, et al. Cambios en la composición corporal en función del grado de demencia en un grupo de ancianos institucionalizados. Nutr Hosp 2013;28(4): 1093-101

20. Camina MA, Barrera S, Domínguez L, et al. Presencia de malnutrición y riesgo de malnutrición en ancianos institucionalizados con demencia en función del tipo y estadío evolutivo. Nutr Hosp 2012;27 (2): 434-40

21. Moral P. Estudio sobre prevalencia de la sarcopenia en una unidad de pacientes crónicos. Recercat Web http://www.recercat.net / bitstream / handle / 2072 / 218125 / TR-MoralMoralPedro.pdf?sequence=1. Accesed 15 november 2013.

22. Johnson Dk, Wilkins Ch, Morris JC. Accelerated weight loss may precede diagnosis in Alzheimer disease. Arch Neurol 2006;63: 1312-7

23. Rolland Y, Czerwinski S, Abellan Van Kan G et al. Sarcopenia: its assessment, etiology, pathogenesis, consequences and future perspectives. J Nutr Health Aging 2008;12: 433-50

24. Burns JM, Johnson Dk, Watts A, Swerdlow RH, brooks WM. Reduced lean mass in early Alzheimer disease and its association with brain atrophy. Arch neurol 2010;67: 428-33

25. Gómez-Cabello A, Vicente Rodríguez G, Vila-Maldonado S, et al. Envejecimiento y composición corporal: la obesidad sarcopénica en España. Nutr Hosp 2012;27(1): 22-30

26. Villalobos Gámez JL, García-Almeida JM, Guzmán de Damas JM et al INFORNUT process: validation of the filter phase-FILNUT-and comparison with other methods for the detection of early hospital hyponutrition. Nutr Hosp 2006;21(4):491-504

27. Barbosa JAP, Rodríguez NG, Hernández YM, et al. Muscle mass, muscle strength, and other functionality components in institutionalized older adults from Gran Caracas-Venezuela. Nutr Hosp 2007;22(5):578-83.

28. Cruz-Jentoft AJ, Triana FC, Gómez-Cabrera MC, et al. La eclosión de la sarcopenia: Informe preliminar del Observatorio de la Sarcopenia de la Sociedad Española de Geriatría y Gerontología. Rev Esp Ger Geron 2011;46(2):100-10 